\title{
Internacionalización en instituciones de Educación Superior: el caso de la Pontificia Universidad Católica de Valparaíso, Chile
}

\section{Internacionalização em instituições de Educação Superior: o caso da Pontifícia Universidade Católica de Valparaíso, Chile}

\author{
Internationalization in institutions of Higher \\ Education: the case of the Pontifical Catholic \\ University of Valparaiso, Chile
}

María Verónica Leiva Guerrero ${ }^{1}$ Hery Segovia Embry ${ }^{1}$

DOI: http://dx.doi.org/10.20435/serie-estudos.v0i0.1408

\begin{abstract}
Resumen: El desarrollo de la Internacionalización en las instituciones de Educación Superior se ha convertido en un aspecto fundamental para abordar las demandas del medio, en un contexto de mayor interacción e integración de países en aspectos económicos, culturales y sociales, instalando progresivamente la dimensión internacional en los distintos espacios del quehacer académico y gestión institucional. El presente estudio tiene como objetivo describir los procesos de internacionalización de la Pontificia Universidad Católica de Valparaíso (PUCV) en lo referido a movilidad estudiantil. Para ello, se desarrolló un estudio documental cuantitativo (BAENA, 1985), recolectándose información a través de análisis de documentos y estadísticas de fuentes internas de la Universidad. Los resultados muestran que la movilidad estudiantil se ha instalado como una de las acciones de internacionalización fundamentales en su Plan de Desarrollo Estratégico, para consolidar la Cooperación Académica Internacional a través de la creación y fortalecimiento de redes de cooperación para la movilidad estudiantil, por medio de los programas de intercambio para cursar un semestre en universidades extranjeras en convenio con la PUCV y de certificación del español como lengua extranjera. Dichas acciones han posicionado a la PUCV como la segunda institución de Educación Superior con mayor movilidad de estudiantes del país. Sin embargo, la cantidad de estudiantes de la PUCV que se movilizan a universidades extranjeras es muy menor en relación a los estudiantes de universidades extranjeras que cursan estudios en la PUCV.
\end{abstract}

Palabras clave: Educación Superior; internacionalización; movilidad estudiantil.

\footnotetext{
${ }^{1}$ Pontificia Universidad Católica de Valparaíso, Valparaíso, Chile.
} 
Resumo: O desenvolvimento da internacionalização nas instituições de Ensino Superior tornou-se um aspecto fundamental para atender às demandas do meio, em um contexto de maior interação e integração dos países nos aspectos econômico, cultural e social, instalando progressivamente a dimensão internacional nos diferentes espaços de trabalho acadêmico e gestão institucional. 0 presente estudo tem como objetivo descrever os processos de internacionalização da Pontifícia Universidade Católica de Valparaíso (PUCV) em relação à mobilidade estudantil. Para isso, foi desenvolvido um estudo documental quantitativo (BAENA, 1985), coletando informações através da análise de documentos e estatísticas de fontes internas da Universidade. Os resultados mostram que a mobilidade estudantil foi instalada como uma das ações fundamentais de internacionalização em seu Plano Estratégico de Desenvolvimento, para consolidar a Cooperação Acadêmica Internacional através da criação e do fortalecimento de redes de cooperação para a mobilidade estudantil, por meio de programas de intercâmbio para cursar um semestre em universidades estrangeiras conveniadas com a PUCV e de certificação do espanhol como língua estrangeira. Essas ações posicionaram a PUCV como a segunda instituição de Ensino Superior com maior mobilidade de estudantes no país. No entanto o número de estudantes da PUCV que se mudam para universidades estrangeiras é muito pequeno em relação aos estudantes de universidades estrangeiras que estudam na PUCV.

Palavras-chave: Educação Superior; internacionalização; mobilidade estudantil.

Abstract: The development of Internationalization in Higher Education institutions has become a fundamental aspect to address the demands of the environment, in a context of greater interaction and integration of countries in economic, cultural and social aspects, progressively installing the international dimension in the different spaces of academic work and institutional management. The objective of this study is to describe the internationalization processes of the Pontifical Catholic University of Valparaíso (PUCV) in relation to student mobility. For this, a quantitative documentary study was developed (BAENA, 1985), collecting information through analysis of documents and statistics from internal sources of the University. The results show that student mobility has been installed as one of the fundamental internationalization actions in its Strategic Development Plan, to consolidate International Academic Cooperation through the creation and strengthening of cooperation networks for student mobility, through exchange programs for a semester in foreign universities affiliated with the PUCV and certification of Spanish as a foreign language. These actions have positioned the PUCV as the second institution of Higher Education with greater mobility of students in the country. However, the number of PUCV students who move to foreign universities is very small in relation to students from foreign universities studying at the PUCV.

Keywords: Higher Education; internationalization; student mobility.

\section{LA INTERNACIONALIZACIÓN EN LA EDUCACIÓN SUPERIOR}

El desarrollo de la Internacionalización en las instituciones de Educación Superior se ha convertido en un aspecto fundamental para abordar las demandas del medio (GAETE; BRATOS, 2012), en un contexto de mayor interacción e integración de los países en aspectos económicos, culturales y sociales, instalando progresivamente la dimensión internacional en los distintos espacios del quehacer académico y gestión institucional. 
Por tanto, la internacionalización se define como un proceso dinámico, dónde se establecen políticas, y se incorporan acciones y procesos que implican una visión internacional al quehacer de las instituciones de Educación Superior, que repercuten, en estas, en su conjunto (CONTRERAS, 2015; GRAF, 2009).

En la contemporaneidad, las discusiones sobre la internacionalización han ganado espacio y se convirtieron en un nuevo desafío para las universidades, las cuales se han visto en la necesidad de adoptar políticas formuladas por organismos nacionales e internacionales, pues estos también hacen las evaluaciones de las instituciones.

Para Stallivieri (2009), el mundo moderno caracterizado por la mercantilización ha puesto a las universidades la posición de que el internacionalismo significa buscar formas de competir con las mejores instituciones de Educación Superior a nivel nacional y mundial.

Otro aspecto que no se puede ignorar que, aunque es un fenómeno importante en el contexto de las instituciones de la Educación Superior, la internacionalización presenta inconsistencias. y que, por lo tanto, no debemos ser ingenuos e irresponsables en consideran que la internacionalización es algo estrictamente positivo, después de todo lo que implica gran responsabilidad social y pedagógica.

Dependiendo del énfasis que las instituciones de Educación Superior otorgan a la cooperación y a la competencia como motores del proceso, la internacionalización puede adoptar un enfoque simbólico (competencia) o transformador (cooperación) (TURNER; ROBSON, 2007). CONTRERAS (2015, p. 170) plantea que "las universidades chilenas conceptualizan y experimentan la internacionalización como un conjunto de actividades que realizan en paralelo con el resto de sus actividades de carácter local y no como un proceso de cambio tendiente a enriquecer su identidad o su quehacer diario".

Dentro de estas actividades de internacionalización de la Educación Superior, que ha experimentado un mayor desarrollo a lo largo del tiempo, es la referida a movilidad estudiantil internacional, como una estrategia para lograr la cooperación académica y la integración regional, además de considerarse como "un elemento clave para la mejora de la formación profesional, la movilización de saberes y la estructuración de redes de intercambio de conocimientos que permitan dar solución a los problemas de los países participantes" (MALDONADO, 2018; SEBASTIÁN, 2004). 
Las estadísticas a nivel internacional, muestran que la movilidad estudiantil internacional ha crecido cinco veces más en los últimos años, entre 2012 y 2017, su número ha pasado de 198 a 220 millones, lo que significa un crecimiento del 10\%, y en 2018 se movilizaron más de 4 millones de estudiantes a nivel mundial (UNESCO, 2019). Es decir, el número de estudiantes en Educación Superior sigue creciendo en el mundo y en la región. En América Latina y el Caribe el aumento ha sido aún más impresionante: de 23,7 a 27,4 millones, con un crecimiento aproximadamente del 16\% en este mismo período (UNESCO, 2019, p. 11).

Los países de América Latina que atraen la movilidad de estudiantes extranjeros son Argentina y Chile, el $84 \%$ y $87 \%$ de los estudiantes extranjeros que reciben, respectivamente proceden de la misma región. El resto de los países acoge fundamentalmente estudiantes extranjeros procedentes de la propia región en porcentajes inferiores. Sin embargo, Ecuador y Brasil no siguen está lógica de movilidad, siendo Brasil el único país donde un poco más de la mitad de sus estudiantes extranjeros (51,5\%) no proceden de la región, sino de Portugal, Estados Unidos, España y de varios países subsaharianos (UNESCO, 2019).

Por otra parte, MALDONADO $(2018$, p. 1) plantea que

[...] la literatura muestra que la movilidad internacional puede disminuir o acrecentar las diferencias sociales, porque quienes tiene posibilidad de tener esas experiencias son estudiantes que tienen recursos económicos y académicos, además de capital social. Pero también la literatura dice que los estudiantes de estratos socioeconómicos no privilegiados que acceden a este tipo de experiencias, pueden tener experiencias muy interesantes $y$ generar un cambio respecto de la forma como aprovechan esas posibilidades. Por ejemplo, puede abrir horizontes educativos que de otra manera no se abrirían.

Complementando lo anterior, MARUM-ESPINOSA (2004, p. 144) señala que [...] la movilidad académica de estudiantes busca mejorar la calidad del desarrollo de recursos humanos y explorar caminos que preparen a los estudiantes para desempeñarse laboral y socialmente en una realidad interdependiente, como ciudadanos responsables de una ciudadanía nacional y mundial.

Por tanto, la movilidad estudiantil internacional, es una muestra de la cooperación educativa en la concurren desde los distintos actores de la Educación Superior esfuerzos y recursos procedentes de diversos frentes que hacen de ella 
un asunto realmente complejo y delicado, puesto que es tanto un reto como una oportunidad para los sistemas educativos contemporáneos (PALMA, 2013).

\section{LA INTERNACIONALIZACIÓN EN LA PONTIFICIA UNIVERSIDAD CATÓLICA DE VALPARAÍSO (PUCV)}

En la Pontificia Universidad de Valparaíso la Dirección de Relaciones Internacionales (DRI) es responsable de profundizar la incorporación de la dimensión internacional, intercultural y global en la formación de pregrado, estudios avanzados, investigación, vinculación con el medio y gestión institucional, lo que permite formar profesionales integrales con una visión del mundo que enriquece el aporte que pueden realizar a la sociedad.

Los esfuerzos institucionales se enfocan en promover las condiciones para el cultivo de los vínculos que actualmente mantiene la institución, así como identificar nuevos espacios de interacción que satisfagan simultáneamente necesidades de interés público y del funcionamiento institucional.

La internacionalización es un ámbito de desarrollo establecido en el Plan de Desarrollo Estratégico de la PUCV. Dicho Plan estratégico propende a una internacionalización integral, cuyos objetivos son los siguientes (PONTIFICIA UNIVERSIDAD CATÓLICA DE VALPARAÍSO [PUCV], 2017):

- Consolidar una cooperación académica internacional que permita fomentar el intercambio de experiencias y de conocimientos, desde una perspectiva global en los distintos ámbitos del quehacer institucional, a saber, formación de pregrado, de estudios avanzados, investigación, vinculación con el medio y gestión.

- Consolidar la movilidad estudiantil para profundizar la incorporación del área internacional en la formación universitaria, considerando el contexto actual de mayor interacción entre países e integración en aspectos productivos, de negocios, culturales y sociales.

\section{COOPERACIÓN ACADÉMICA INTERNACIONAL}

En Cooperación Académica Internacional se fomentará la generación de redes de cooperación. Para ello se incentivará el uso de los convenios vigentes y se procurará la creación de nuevas alianzas. A su vez, se promoverá la movilidad de 
académicos y se apoyará, tanto a nivel de pregrado como de estudios avanzados, el establecimiento de convenios de doble grado/título y de codirección de tesis, respectivamente. En tales actividades se buscará propiciar la participación de las Unidades Académicas, especialmente de aquellas que muestran niveles iniciales en materia de desarrollo de actividades internacionales.

Además, se incrementará los esfuerzos para difundir las actividades y resultados de la internacionalización entre la comunidad PUCV. Para ello, se trabajará en coordinación con las áreas a cargo del pregrado y de los estudios avanzados e investigación, de manera de comunicar los logros alcanzados, buscando sinergias que permitan replicarlos y ampliar sus alcances.

\section{MOVILIDAD ESTUDIANTIL EN LA PONTIFICIA UNIVERSIDAD CATÓLICA DE VALPARAÍSO}

En movilidad estudiantil, se han implementado acciones para atraer más estudiantes extranjeros a programas regulares de pregrado y de estudios avanzados. Junto con ello, la Universidad ha aumentado la cobertura de sus programas de intercambio y de aquellos que son especialmente diseñados para alumnos internacionales. Asimismo, se han fortalecido programas de enseñanza y certificación del español como lengua extranjera.

También se ha incrementado el intercambio de estudiantes de la Pontificia Universidad Católica de Valparaíso. Para ello se han revisado y perfeccionado los mecanismos, tanto procedimentales como reglamentarios, para facilitar que alumnos de pregrado de la Universidad realicen experiencias de intercambio. Paralelamente, se ha facilitado la interacción entre estudiantes nacionales y extranjeros en Chile.

Dichos procedimientos implican la gestión de un Convenio Específico de Movilidad Estudiantil por las distintas Unidades Académicas, que tiene como propósito realizar actividades de intercambio estudiantil, en pregrado y/o postgrado, de manera recíproca y definiendo condiciones, contenidos, recursos, y modalidades operativas de implementación.

Para la realización de los convenios, la Pontificia Universidad Católica de Valparaíso tiene definidas las siguientes políticas:

1. Todos los Convenios nacionales e internacionales de Cooperación 
Académica deben ser gestionados por la Dirección de Relaciones Internacionales (DRI).

2. El Director de la Unidad Académica interesada en formalizar un convenio debe solicitar por escrito a la Vicerrectoría de Desarrollo la firma del convenio con la Institución de Educación Superior que quiere estrechar vínculos.

3. Una realizado todo el proceso y elaborado el documento "Convenio Específico" el Rector, único responsable, firma dicho convenio.

4. La vigencia de los Convenios Específicos de Movilidad Estudiantil será consensuada por las partes. Generalmente, estos convenios tienen una vigencia de 4 años con renovación automática por periodos iguales y sucesivos.

Todos los convenios, sin excepción, deben contener las cláusulas relativas a: Coordinadores, Propiedad Intelectual, Resolución de conflictos y procedimientos.

La PUCV a la fecha tiene convenios formalizados con más de 300 universidades del mundo. Los estudiantes de la universidad (PUCV) tienen la posibilidad de cursar un semestre en el extranjero y, en algunos casos, programas de doble titulación. Estas actividades académicas en el extranjero, se pueden realizar a través de los siguientes convenios:

- Convenios Bilaterales: la PUCV mantiene más de 40 convenios con universidades de Alemania, Australia, Canadá, Corea, Costa Rica, España, Estados Unidos, Francia, Italia, México, Portugal, Rumania y Suecia.

- ISEP: la PUCV es miembro del International Student Exchange Program, que permite a sus alumnos asistir a más de 100 universidades en Estados Unidos y 130 en el resto del mundo.

- DAAD: programa que permite a alumnos de Ingeniería y Agronomía de la PUCV hacer una estadía de un año en prestigiosas universidades alemanas, con financiamiento parcial del Servicio Alemán de Intercambio Académico (DAAD).

- CINDA: a través de un convenio con el Centro Interuniversitario de Desarrollo, las universidades más importantes de Latinoamérica abren sus puertas para que los estudiantes de la PUCV puedan realizar estancias académicas de un semestre. 
Por último, se han promovido acciones focalizadas de difusión de la Pontificia Universidad Católica de Valparaíso en el extranjero, especialmente, dando a conocer los programas de pregrado y de estudios avanzados. Actualmente, cuenta con la Learn Chile, plataforma destinada a difundir toda la oferta de pregrado y postgrado en el extranjero.

Considerando lo anterior, El presente artículo tiene como objetivo describir los procesos de internacionalización de la Pontificia Universidad Católica de Valparaíso (PUCV) en las áreas de cooperación académica internacional y movilidad estudiantil. Para ello, se desarrolló un estudio documental cuantitativo (Baena, 1985), recolectándose información a través de análisis de documentos, estadísticas de fuentes internas de la universidad, como también de información verbal entregada por directivos y encargados responsables de los procesos de cooperación y movilidad internacional.

\section{METODOLOGÍA}

Se realizó una investigación documental cuantitativa (BAENA, 1985) que consideró como objetivo principal la cuantificación información emanada de material impreso: Reportes de Sostenibilidad de la PUCV 2016, 2017 y electrónico: Bases de Datos de la Dirección de Relaciones Internacionales de la Pontificia Universidad Católica de Valparaíso, sobre las variables movilidad estudiantil y docente entre los años 2016 y 2018. Con dicha información procedió a su sistematización y procesamiento estadístico en función de los siguientes variables e indicadores:

\subsection{Movilidad de Estudiantes:}

a) Cantidad de estudiantes extranjeros de intercambio en la PUCV y cantidad de estudiantes de la PUCV de intercambio en el extranjero.

b) Procedencia (País) de estudiantes de intercambio extranjeros en la PUCV.

c) País destino de estudiantes PUCV de intercambio.

d) Área disciplinar de estudios de los estudiantes extranjeros en la PUCV.

e) Facultad de origen de los estudiantes PUCV que cursan en el extranjero. 


\section{RESULTADOS}

\subsection{Movilidad de Estudiantes}

A continuación, se presentarán los resultados de movilidad de estudiantes de acuerdo a indicadores definidos en apartado metodología.

a) Cantidad de estudiantes extranjeros de intercambio en la PUCV y cantidad de estudiantes de la PUCV de intercambio en el extranjero.

Respecto de la movilidad de estudiantes entre los años 2016-2018, podemos observar en Tabla 1, que existe mayor movilidad, 59\% más, de estudiantes extranjeros hacia la PUCV, que de estudiantes PUCV a universidades extranjeras.

Tabla 1 - Cantidad de estudiantes extranjeros de intercambio en la PUCV y cantidad de estudiantes de la PUCV de intercambio en el extranjero

\begin{tabular}{lcccc}
\hline & $\mathbf{2 0 1 6}$ & $\mathbf{2 0 1 7}$ & $\mathbf{2 0 1 8}$ & $\begin{array}{c}\text { Total, período } \\
\mathbf{2 0 1 6 - 2 0 1 8}\end{array}$ \\
\hline Número de estudiantes extranjeros en PUCV & 759 & 788 & 817 & 2364 \\
Número de estudiantes PUCV en el extranjero & 222 & 190 & 196 & 608 \\
\hline
\end{tabular}

Fuente: Dirección de Relaciones Internacionales (2019).

b) Procedencia (País) de estudiantes de intercambio extranjeros en la PUCV.

La mayor cantidad de estudiantes extranjeros con movilidad en la PUCV, tienen como país de procedencia Estados Unidos, esto corresponde al 53, 3\%, en relación a los otros países de procedencia de los estudiantes. Solo un $1 \%$ de los estudiantes extranjeros con movilidad en la PUCV provienen de los países China y Japón. Ver Tabla 2. 
Tabla 2 - Procedencia (País) de estudiantes de intercambio extranjeros en la PUCV, 2016-2018

\begin{tabular}{lcc}
\hline \multicolumn{1}{c}{$\begin{array}{c}\text { País de origen de los } \\
\text { estudiantes }\end{array}$} & $\begin{array}{c}\text { Cantidad } \\
\text { de estudiantes }\end{array}$ & $\begin{array}{c}\text { Proporción del total } \\
\text { de estudiantes }\end{array}$ \\
\hline Estados Unidos & 1.259 & $53,3 \%$ \\
España & 267 & $11,3 \%$ \\
Francia & 180 & $7,6 \%$ \\
Alemania & 98 & $4,1 \%$ \\
Italia & 76 & $3,2 \%$ \\
Colombia & 73 & $3,1 \%$ \\
México & 64 & $2,7 \%$ \\
Portugal & 33 & $1,4 \%$ \\
Bélgica & 28 & $1,2 \%$ \\
Japón & 26 & $1,1 \%$ \\
China & 25 & $1,1 \%$ \\
Otros países & 235 & $9,9 \%$ \\
TOTAL & 2.364 & $100 \%$ \\
\hline
\end{tabular}

Fuente: Dirección de Relaciones Internacionales (2019).

c) País de destino de estudiantes PUCV de intercambio

Los estudiantes de la PUCV, en su movilidad al extranjero prefieren mayoritariamente universidades de países de Europa, siendo España el país de preferencia, después Francia, Italia y Alemania. La preferencia mayoritaria de intercambio en España ( $N=222)$, de acuerdo a lo declarado por el coordinador de intercambio de la PUCV, se debe al idioma. Ver Tabla 3. 
Tabla 3 - Cantidad de estudiantes de intercambio de la Pontificia Universidad Católica de Valparaíso en el extranjero, por país de destino, 2016-2018

\begin{tabular}{lcc}
\hline \multicolumn{1}{c}{ País de destino } & Total estudiantes & Proporción sobre el total \\
\hline España & 222 & $36,5 \%$ \\
Francia & 88 & $14,5 \%$ \\
Italia & 64 & $10,5 \%$ \\
Alemania & 47 & $7,7 \%$ \\
Estados Unidos & 44 & $7,2 \%$ \\
Canadá & 26 & $4,3 \%$ \\
Brasil & 24 & $3,9 \%$ \\
Irlanda & 22 & $3,6 \%$ \\
Portugal & 14 & $2,3 \%$ \\
Colombia & 11 & $1,8 \%$ \\
México & 10 & $1,6 \%$ \\
Holanda & 6 & $1,0 \%$ \\
Otros & 30 & $4,9 \%$ \\
Total & 608 & $100 \%$ \\
\hline
\end{tabular}

Fuente: Dirección de Relaciones Internacionales (2019).

Al analizar las tablas 1 y 2 , observamos que el número de estudiantes de la PUCV que se movilizan a universidades extranjeras es muy menor en relación a los estudiantes extranjeros que llegan a la PUCV, esto puede tener su respuesta en las dificultades socioeconómicas: la mayoría de estudiantes no cuenta con suficientes medios para enfrentar los gastos asociados a la Movilidad Estudiantil. d) Área disciplinar de estudios de los estudiantes extranjeros en la PUCV

En relación al interés del área disciplinar de estudios que los estudiantes extranjeros optan en la PUCV, corresponden en mayor medida a Ciencias Sociales y Humanidades (20\%), otros (20\%), Ingeniería y Literatura (13\%), y el área de menor interés por cursar en la PUCV, es Educación (5\%). Ver Figura 1. 
Figura 1 - Área disciplinar de estudios de los estudiantes extranjeros en la PUCV. Cantidad de estudiantes y proporción del total en intercambio Período 2016-2018

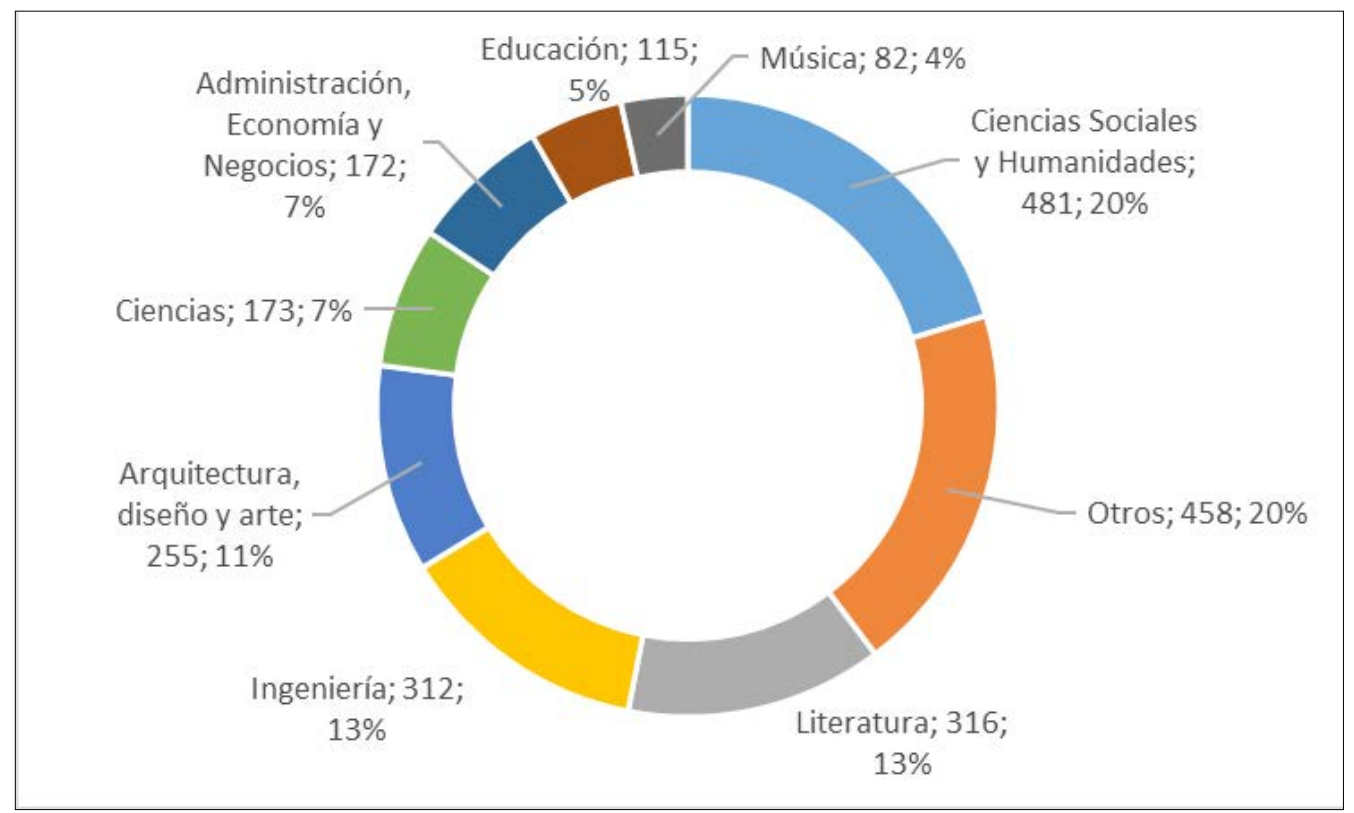

Fuente: Dirección de Relaciones Internacionales (2019).

e) Facultad de origen de los estudiantes PUCV que cursan en el extranjero

A diferencia de las áreas disciplinares de estudio que cursan los estudiantes extranjeros en la PUCV, donde Ingeniería es la menos cursada, en el caso de las áreas disciplinares de estudiantes de la PUCV que tienen movilidad en el extranjero, la Facultad de Ingeniería, como se observa en la figura 2, tiene el porcentaje más alto (30\%) de estudiantes con movilidad en universidades extranjeras. 
Figura 2 - Facultad de origen de los estudiantes PUCV en intercambio en el extranjero. Cantidad de estudiantes y proporción del total en intercambio Período 2016-2018

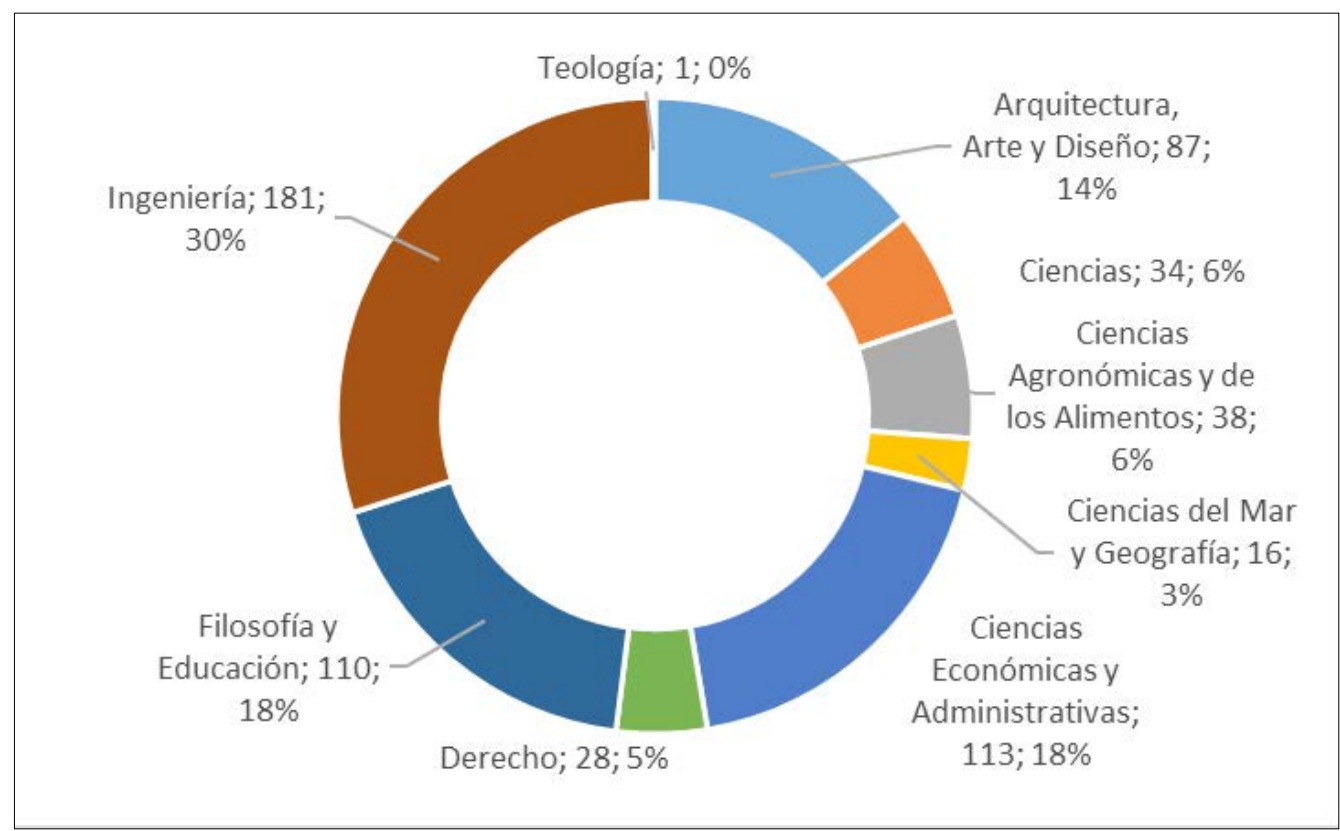

Fuente: Dirección de Relaciones Internacionales (2019).

\section{CONCLUSIONES}

La difusión de actividades de internacionalización dentro de las unidades académicas ha logrado proyectar a la Pontificia Universidad Católica de Valparaíso, en todo el mundo, como también con organismos internacionales y agencias de cooperación. Además, ha encabezado los esfuerzos de la marca sectorial para servicios de Educación Superior, Learn Chile, plataforma destinada a difundir toda la oferta de pregrado y postgrado en el extranjero. Dichas acciones han ubicado a la PUCV, como la segunda institución de Educación Superior con mayor movilidad de estudiantes del país. No obstante, se debe seguir potenciando la movilidad de estudiantes de la PUCV a universidades extranjeras, y la movilidad de estudiantes extranjeros a las áreas de educación de la PUCV, que tienen más bajos índices de movilidad. 
Para lo anterior, y considerando las políticas de cooperación académica y movilidad de estudiantes, las unidades académicas deben idear y gestionar estrategias para propender a una mayor movilidad al extranjero de sus estudiantes, toda vez que la movilidad estudiantil es uno de los aspectos más dinámicos de la internacionalización de las universidades, y se constituye como un elemento fundamental del conjunto de políticas educativas en materia de internacionalización de la Educación Superior y colaboración en materia de desarrollo (PALMA, 2013).

Finalmente, es preciso señalar que entre los años 2016 y 2018 la Pontificia Universidad Católica de Valparaíso estableció 81 nuevos convenios de cooperación académica internacional, con 20 países diferentes, permitiendo sumar nuevos destinos para intercambio de estudiantes y académicos, así como convenios de doble titulación con prestigiosas universidades. No obstante, las evidencias arrojadas por este estudio nos permiten concluir que la PUCV ha adoptado un enfoque simbólico (competencia), no logrando aún ser una institución internacionalizada, más bien, es una universidad que realiza actividades académicas internacionales.

\section{REFERENCIAS}

BAENA, G. Instrumentos de Investigación. México: Editores Mexicanos Unidos, 1985.

CONTRERAS, P. Conceptualización y experiencia de la internacionalización del pregrado chileno. Calidad en la educación, Santiago, n. 43, p. 169-200, dic. 2015. Disponible en: https://scielo.conicyt.cl/scielo.php?script=sci_arttext\&pid=S071845652015000200006\&lng=es\&nrm=iso. Acceso el: 9 jul. 2019. Doi: http://dx.doi. org/10.4067/S0718-45652015000200006

DIRECCIÓN DE RELACIONES INTERNACIONALES. Pontificia Universidad Católica de Valparaíso. Valparaíso, 2019.

GAETE QUEZADA, R; BRATOS MARTIN, M. Una mirada a la internacionalización universitaria desde la perspectiva de la responsabilidad social: discursos de los jóvenes investigadores. Estudios Pedagógicos, Valdivia, v. 38, n. 1, p. 255-72, 2012. Disponible en: https://scielo.conicyt.cl/scielo.php?script=sci_arttext\&pid=S071807052012000100015\&lng=es\&nrm=iso. Acceso el: 9 jul. 2019. Doi: http://dx.doi. org/10.4067/S0718-07052012000100015 
Internacionalización en instituciones de Educación Superior: el caso de la Pontificia Universidad Católica de Valparaiso, Chile

GRAF, L. Applying the varieties of capitalism approach to higher education: comparing the internationalization of german and british universities. European Journal of Education, v. 44, n. 4, p. 569-585, nov. 2009.

MALDONADO, A. La movilidad internacional puede disminuir o acrecentar las diferencias sociales. Centro de Investigación Avanzada en Educación. Notícias. Santiago, jun. 2018. Disponible en: http://www.ciae.uchile.cl/index.php?page=view_ noticias\&langSite=es\&id=1387\&externo=boletin. Acceso el: 9 jul. 2019.

MARUM-ESPINOSA, E. La movilidad de estudiantes, características y opiniones de los estudiantes extranjeros en Guadalajara, Jalisco, México. Perfiles educativos, México, v. 26, n. 105/106, p. 143-58, enero 2004. Disponible en: http://www.scielo.org.mx/scielo. php?script=sci_arttext\&pid=S0185-26982004000100007\&lng=es\&nrm=iso. Acceso el: 8 dic. 2019.

PALMA, J. J. G. Movilidad Estudiantil internacional y cooperación educativa en el nivel superior de educación. Revista Iberoamericana de Educación, n. 61, p. 59-76. 2013.

PONTIFICIA UNIVERSIDAD CATÓLICA DE VALPARAÍSO. Plan de Desarrollo Estratégico, 2017-2022. Valparaíso: PUCV, 2017. Disponible en: https://www.pucv.cl/pucv/site/ artic/20170614/asocfile/20170614212256/pde_2017_cnt.pdf. Acceso el: 9 jul. 2019.

SEBASTIÁN, J. Cooperación e internacionalización de las universidades. Buenos Aires: Biblos, 2004.

STALLIVIERI, L. As dinâmicas de uma nova linguagem intercultural na mobilidade acadêmica internacional. Orientador: Héctor Valencia. 2009. Tese (Doutorado em Línguas Modernas) - Universidad del Salvador, Buenos Aires, Argentina, 2009.

TURNER, Y.; ROBSON, S. Competitive and cooperative impulses to internationalization: reflecting on the interplay between management intentions and the experience of academics in a British University. Education, Knowledge and Economy, v. 1, n. 1, p. 65-82, abr. 2007. Doi: http://dx.doi.org/10.1080/17496890601128241

UNESCO. La movilidad en Educación Superior en América Latina y el Caribe: retos y oportunidades de un convenio renovado para el reconocimiento de estudios, títulos y diplomas. Caracas: IESALC, 2019. 


\section{Sobre los autores:}

María Verónica Leiva Guerrero: Postítulo en Procesos Pedagógicos Mención Gestión Curricular por la Universidad de Santiago de Chile. Doctora em Didáctica em Ciencias de la Educación por la Universidad de Oviedo, España. diplomada en Proyectos Educacionales por la Universidad Marítima de Chile. Actualmente es Profesora Adjunta, Jornada Completa de la Escuela de Pedagogía y Directora del Magíster em Liderazgo y Gestión em Organizaciones Escolares em la Pontificia Universidad Católica de Valparaíso. Profesora de Educación Básica por la Universidad de Playa Ancha. E-mail: veronica.leiva@pucv.cl, Orcid: https://orcid.org/0000-0002-7641-0087

Hery Segovia Embry: Ingeniero Comercial de la Pontificia Universidad Católica de Valparaíso. Magíster em Psicología Organizcaional de la Universidad Adolfo Ibáñez, Magíster em Dirección de Empresas del Institute for Executive Development (IEDE). Profesor agregado de la Escuela de Negocios y Economía y Profesor del núcleo del Programa de Magíster en Gestión y Liderazgo de Organizaciones Escolares de la Pontificia Universidad Católica de Valparaíso. Gerente de la Fundación Educacional Rector Rubpen Castro de la Pontificia Universidad Católica de Valparaíso. E-mail: hery.segovia@pucv.cl Orcid: https://orcid.org/0000-0002-5045-425X

\section{Recibido el 9 de diciembre de 2019}

Aprobado el 14 de febrero de 2020 